\title{
Effect on Microstructure and Microhardness of Equiatomic NiCoAlFeMoTi High Entropy Alloys Produced by Mechanical Alloying and Subsequent Arc-Melting
}

\author{
F.J. Baldenebro-Lopez ${ }^{1,2,}$, C.D. Gómez-Esparza1 ${ }^{1}$, J.A. Baldenebro-Lopez ${ }^{2}$, I. Estrada-Guel ${ }^{3}$, J.T. \\ Elizalde-Galindo ${ }^{1}$, H. Camacho-Montes ${ }^{1}$, and R. Martínez-Sánchez ${ }^{3}$ \\ 1. IIT-Universidad Autónoma de Ciudad Juárez., 32310 Ciudad Juárez, Chih., México. \\ 2. FIM-Universidad Autónoma de Sinaloa, 81223 Los Mochis, Sinaloa, México. \\ 3. Centro de Investigación en Materiales Avanzados (CIMAV), 31109 Chihuahua, Chih., México.
}

High entropy alloys (HEAs) have been known as a new type of material with unusual properties. HEAs contain at least five major elements in equiatomic or near-equiatomic proportions [1], and in general tend to form simple FCC and/or BCC microstructures in form of solid solutions (SS) [2]. Several chemical systems in HEAs have been investigated, but Mo-Ti couple has been rarely reported. The aim of this work was to characterize the microstructure of HEA with Mo-Ti produced by mechanical alloying (MA) and the effect of arc melting on NiCoAlFeMoTi alloy.

The HEAs have been fabricated by different routes. The melting process has become the preferred technique to produce these alloys. It is well known that samples produced by MA followed by consolidation, possess a higher pore density than samples fabricated by casting. However, the melting route leads to the segregation problem, while in the MA process, homogenous chemical distribution and solid solubility extension can be reached. However, in the field of HEAs, the synthesis of new alloys attempts to solve the limitations of traditional metallurgy techniques. With the aim to consider alternative routes to synthesize high entropy alloys and improve their performance, in this work the simultaneous effect of mechanical alloying and arc melting on the fabrication of an AlCoFeMoNiTi alloy was studied.

Elemental powders with purity higher than $99.5 \%$ were used as raw materials to synthesize equiatomic NiCoAlFeMoTiCr HEA. The MA was performed in a high-energy ball mill (SPEX-8000M) for a time of $10 \mathrm{~h}$, under an argon atmosphere. Methanol was used as a process control agent to avoid metal agglomeration. The milled powders were mechanically pressed into cylindrical samples with $10 \mathrm{~mm}$ in diameter under $1.5 \mathrm{GPa}$ of pressure, using a uniaxial compaction die in a hydraulic press and subsequent melting by arc under an argon atmosphere to prevent oxidation. The ingots were remelted at least 5 times to improve chemical homogeneity.

In the Figure 1 a DF-TEM micrograph of a single powder particle after milling is presented, it was observed the nanocrystals presence and the EDS-TEM results showed a near-equiatomic composition in powders after $10 \mathrm{~h}$ of milling. Figure 2 shows SEM images of a representative microstructure after arc melting. In order to determine whether the effect of the mechanical alloying is kept after melting, the ascast samples from un-milled (Figure 2a) and milled powders (Figure 2b) are shown. Both samples exhibit the formation of similar phases, a gray phase (Ni-Co-Ti), a continuous bright phase (Fe-Mo-Co), a discontinuous bright phase (Mo) and a dark phase (Ti); this effect is contrary than expected by traditional metallurgy where the effect of mechanical alloying after melting is noticeable by a more uniform distribution of phases and their reduction in size. Z-contrast TEM image of the as-cast sample of Figure 3 shows the presence of same phases detected in the Figure 2, which was determined by EDS/SEM analysis. The results of microhardness test are shown in Figure 4. The highest hardness values were achieved for the as-cast sample from milled powder $(767 \mathrm{HV})$, followed by as-cast sample from un-milled $(652 \mathrm{HV})$. According to the hardness, it can be corroborated that the effect of 
mechanical alloying in the enhancement of mechanical performance is maintained after melting.

A nanocrystalline AlCoFeMoNiTi alloy was successfull synthesized by mechanical alloying. Consolidated samples by arc melting presented different microstructures respect to grain size and according to electron microscopy observations, both alloys exhibit the formation of two main phases which are similar in chemical composition. The microhardness results showed that the sample made with milled powder reached the highest hardness.

References:

[1] Y.L. Chen, Y.H. Hu, C.W. Tsai, C.A. Hsieh, S.W. Kao, J.W. Yeh, T.S. Chin and S.K. Chen, J. Alloys Compd. 477 (2008) p. 696.

[2] T.T. Shun and Y.C. Du, J. Alloys Compd. 479 (2009) p. 157.

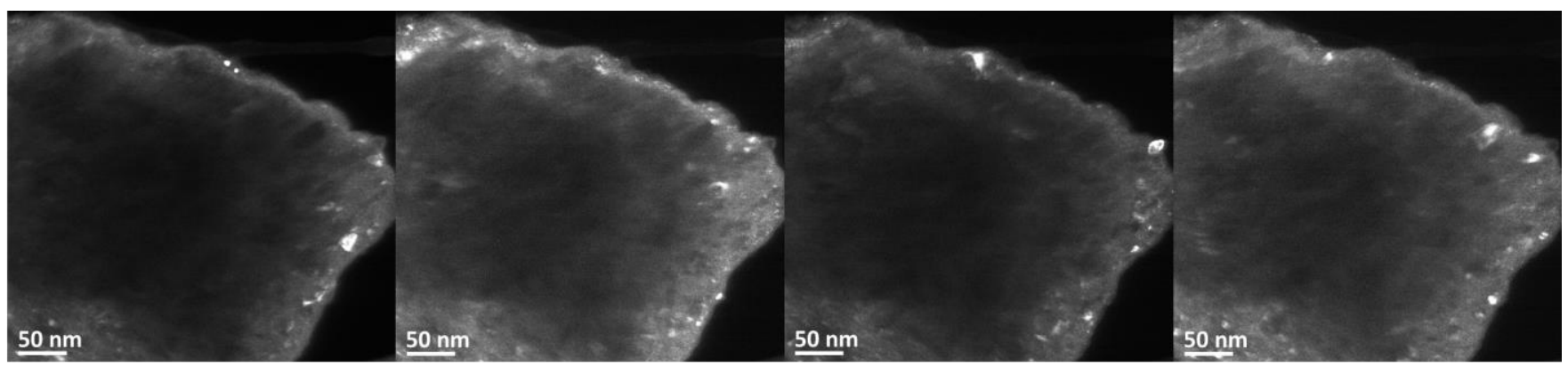

Figure 1. TEM micrographs of NiCoAlFeMoTi powder alloy after $10 \mathrm{~h}$ of milling.

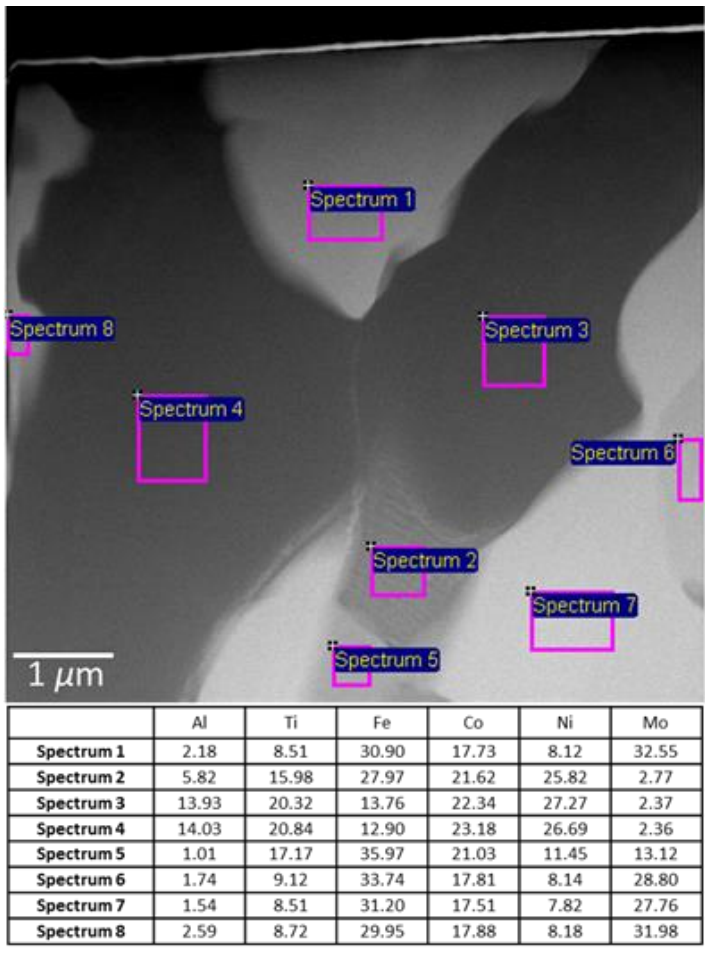

Figure 3. Microstructure images of the sample Z-contrast TEM (STEM mode).

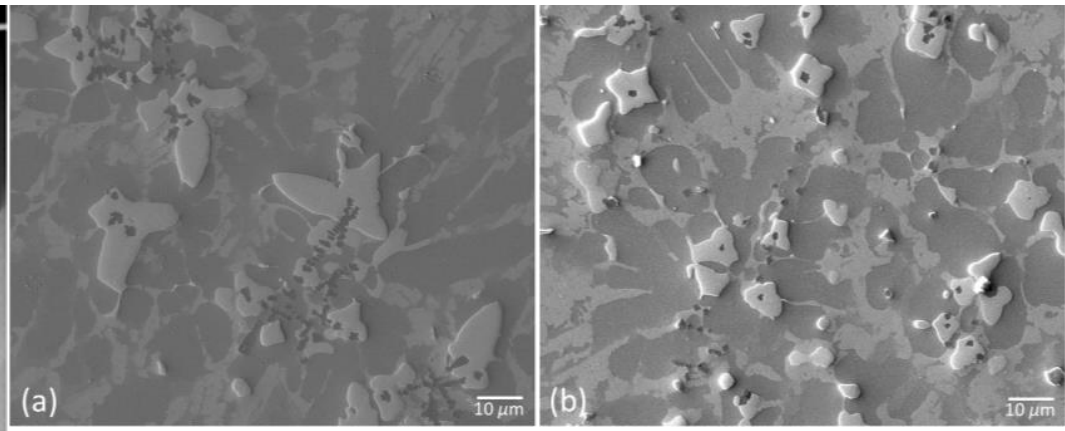

Figure 2. SEM images of the as-cast samples: (a) un-milled and (b) milled powders.

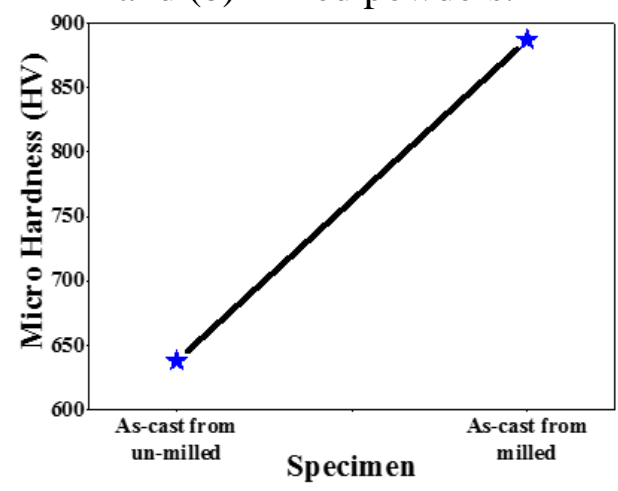

Figure 4. SE-SEM of AlCoNi reinforced with SiC. 